\title{
Apuntes hacia una crítica cuir del sistema de educación pública en Costa Rica (parte II) ${ }^{1}$
}

\author{
Luis Alonso Rojas-Herra* \\ https://orcid.org/0000-0002-3503-4214
}

\section{Resumen}

Este ensayo ${ }^{2}$ es un intento por incluir saberes epistémicos y etimológicos producidos desde el pensamiento de las vanguardias feministas y de la teoría cuir, que tradicionalmente han sido excluidos de la corriente de pensamiento hegemónico ${ }^{3}$. La historia de la construcción del Estado-nación y la institucionalidad se ha narrado, de manera favorable, desde una perspectiva del privilegio blanqueado, heterosexualizado y capitalista ${ }^{4}$, lo que excluye e invisibiliza otras formas de producción de pensamiento contestatarias a las tradicionales.

Palabras clave: Epistemología cuir, etimología cuir, pedagogía cuir, reforma educativa.

\begin{abstract}
* Licenciado en Arquitectura de la Universidad Veritas, de Costa Rica. Actualmente cursa el posgrado en Paisajismo y Diseño de Sitio en la Universidad de Costa Rica. Investigador del Centro de Investigación, Cultura y Desarrollo (CICDE) de la Universidad Estatal a Distancia, de Costa Rica. Docente universitario en varios centros educativos privados. Ganador del segundo lugar en la categoría investigación de la Bienal Centroamericana de Paisaje. Correo: lrojash@uned.ac.cr
\end{abstract}

\footnotetext{
${ }^{1}$ Esta propuesta ensayística se divide en dos partes: Un primer artículo con reflexiones teóricas que incorpora saberes disidentes en la línea de pensamiento hegemónico; el cual se publicó en la revista Disenso, de la Universidad Nacional de San Agustín de Arequipa, en Perú. Mientras que esta segunda parte corresponde a un ensayo de carácter propositivo, donde teóricamente se abordan tres reformas estructurales e interseccionales en la agenda del sistema educativo, para contribuir con una transición más sana a un sistema educativo inclusivo y horizontal con verdadero poder de transformación social. ${ }^{2}$ Aprobado por el comité editorial de la Revista Espiga en sesión del 14 de mayo de 2021. La sección en que se publica no es arbitrada mediante el proceso de pares.

${ }^{3}$ Miriam Solá y Elena Urko, Transfeminismos epistemes, fricciones y flujos (Navarra: Ediciones

Txalaparta, 2018).

${ }^{4}$ Ibíd.
} 


\section{Breve introducción al sistema educativo como régimen fundacional del Estado}

La educación es uno de los regímenes fundacionales de la construcción del Estadonación. En el 2021, en la región, se celebran los 200 años desde que inició el proceso de constitución de un territorio independiente. Esta introducción es una breve explicación del contexto en que se construyó ese mito fundacional de la educación en distintos momentos históricos del periodo de las últimas 20 décadas.

La educación organizada, homogénea, con características etnocentristas y patriarcales, siempre ha sido uno de los objetivos principales de la transición de la época colonial a la construcción de un Estado nacionalista independiente y occidentalizado $^{5}$. Con la colonización se dio paso, de manera paulatina, a los primeros rasgos educativos de una región oprimida por los conquistadores y de un territorio en proceso de occidentalización. La educación durante la época colonial tuvo como principal intención el educar a los criollos (hijos de los españoles que nacían en América) y evangelizar a la población en general, criollos e indígenas, estos últimos considerados bestias incultas por los españoles, desde una perspectiva jerárquica.

Por ello, la presencia del clérigo europeo durante la colonización le permitió ejercer poder legitimado por políticas de orden público, como la campaña de instrucción a los indígenas de 1565 , guiada por religiosos ${ }^{6}$, que pretendía homogenizar a la población bajo un modelo educativo exportado por los colonizadores españoles, que eliminó, de manera violenta, casi por completo a todas las formas de enseñanza de los pueblos indígenas originarios de la región o sociedades autóctonas costarricenses, denominado como modelo de educación espontánea de carácter intuitivo ${ }^{7}$.

Este periodo se caracterizó por la inestabilidad política de la región, que incidía en el acceso a los recursos para el financiamiento de la educación, materializado en la falta de infraestructura arquitectónica. En este periodo la educación se ejercía en centros comunitarios o espacios eclesiásticos. No es hasta el siglo XVIII que la monarquía española financia los primeros centros educativos en la región centroamericana; sin embargo, en Costa Rica, el primer centro educativo escolar se funda por los esfuerzos de los clérigos y la movilidad social en Cubujuquí de Heredia, en $1738^{8}$. En este punto, es importante aclarar el rol que tuvieron los movimientos sociales en la construcción de un sistema educativo ciudadano durante todos los periodos de la cronología de la educación costarricense, como se retomará más adelante.

Durante los primeros años, después de la colonización y hacia la transición de la fundación de la república, la educación se convirtió en una prioridad de la administración pública, sobre todo para las municipalidades, quienes fueron las encargadas de la inspección de la educación en este periodo. Sin embargo, existían pocos instrumentos jurídicos o normativas para la instrucción ciudadana de los

\footnotetext{
${ }^{5}$ Bernal Martínez Gutiérrez, Cronología de la educación costarricense (Costa Rica: Imprenta Nacional, 2016), 9.

${ }^{6}$ Ibíd., 14.

${ }^{7}$ Ibíd., 11.

${ }^{8}$ Ibíd., 16.
} 
habitantes de la época y, los que existían, tenían una fuerte influencia y perspectiva del discurso cristiano, como lo fue el catecismo político de $1822^{9}$.

Durante el siglo XIX, en los primeros años de la fundación de la república, el fenómeno educativo estuvo marcado por un periodo de incertidumbre política, caracterizado, entre otros aspectos, por dar acceso a la educación a un grupo privilegiado de la sociedad costarricense. En este periodo, y durante más de 78 años, se desarrollaron distintos hitos educativos que constituirán las cimentaciones para la gran reforma educativa del siglo $\mathrm{XX}^{10}$. Entre algunos de esos hitos se encuentran: la creación de los primeros conatos de normativa como la ley de compulsión escolar en $1832^{11}$, la construcción de la primera casa de enseñanza de Santo Tomás en $1824^{12}$, la primera imprenta en $1830^{13}$, se otorga a la educación pública un marco legal constitucional, en $1844^{14}$, y La carta fundamental de $1869^{15}$.

Todos estos hitos educativos juegan un rol significativo que dan paso a la primera gran reforma estructural educativa de 1900. Esta reforma estuvo marcada por una fuerte influencia colonial imperialista del conocimiento, que la Ilustración y las políticas liberales estaban generando en Europa occidenta ${ }^{16}$. Estas corrientes occidentalizadas influyeron considerablemente en la constitución de un sistema educativo moderno, formalizado e institucionalizado para el país. Este modelo siguió manteniendo las mismas características fundacionales del periodo colonial, sobre un acceso elitista que priorizaba una clase social privilegiada, reproduciendo un modelo pedagógico colonial sobre el conocimiento, así como un modelo jerárquico de su estructura organizacional.

Sin embargo, y gracias al poder, la de movilidad social de distintos grupos de ciudadanos que desean validar su derecho a la educación y ante la ausencia de esta, durante el periodo de la construcción del Estado benefactor en $1957^{17}$ (la gran alianza pública, privada y sociedad civil en benéfico de los más empobrecidos) se logra la segunda reforma estructural educativa del país con la Ley Fundamental de Educación (ley N $\mathrm{N}^{\mathrm{O}} 2160$ ).

Esta ley establece todos los parámetros para la administración de la educación pública, tal y como se conoce hoy. A pesar de que en su momento la propuesta de ley fue innovadora, hasta la actualidad solo ha tenido 11 propuestas aprobadas de reforma en las últimas cinco décadas, entre 1962 y 2014, que la institucionalidad llama hitos normativos ${ }^{18}$. De estas, solo una ha correspondido a una propuesta de reforma de carácter estructural para el sistema educativo público, siendo esta la ley de la reforma de la escuela rural de 1966.

Actualmente, los Estados soberanos modernos hetero-capitalistas occidentalizados se enfrentan a un conflicto emergente de las últimas décadas, la privatización de la

\footnotetext{
${ }^{9}$ Ibíd., 19.

${ }^{10}$ Ibíd., 22.

${ }^{11}$ Ibíd., 19.

12 Ibíd.

${ }^{13}$ Ibíd.

14 Ibíd., 20.

${ }^{15}$ Ibíd.

${ }^{16}$ Ibíd., 22.

${ }^{17}$ Ibíd., 29.

${ }^{18}$ Programa Estado de la Nación, Sexto Informe Estado de la Educación (San José: Servicios Gráficos, A. C., 2017), recuperado de https://www.estadonacion.or.cr/educacion2017/assets/ee6informe-completo.pdf
} 
educación, como un privilegio de la clase social, poniendo en peligro el derecho a ella. Este texto pretende descolocar algunos regímenes fundacionales en la construcción del mito de la educación imperante en el discurso nacionalista oficial, pero que contrasta radicalmente con la realidad precarizada de la región. Por otro lado, el texto no pretende dar soluciones concretas, al contrario, la intención es hacer señalamientos epistémicos y etimológicos de situaciones que se deben visibilizar desde una perspectiva decolonial, militante y cuir.

\section{Devenir hacia una lectura interseccional del sistema educativo público}

Dentro de la coyuntura actual del país como Estado soberano moderno heterocapitalista occidentalizado, el sistema educativo público enfrenta diversas variables, muchas de ellas emergentes (como la crisis sanitaria producida por la pandemia del Covid-19) y otras de carácter histórico que imposibilitan seguir sosteniendo el discurso nacionalista (romántico e idealista) que por décadas ha alimentado al ciudadano costarricense sobre la percepción de un sistema de educación pública costarricense universalizado (robusto y equitativo).

La principal crítica al sistema educativo nacionalista es hacia su forma de organización constituida de manera jerárquica, la cual promueve valores patriarcales ${ }^{19}$ como el respeto, honor, reconocimiento, valorización y mejores oportunidades a aquellos privilegiados que ejercen prácticas hetero-capitalistas sobre grupos o personas menos privilegiadas. Su compleja estructura jerárquica organizativita del sistema constituye el principal obstáculo para la transformación en tres ejes o reformas significativas e interseccionales que se van a desarrollar en este texto; se establece con el fin de lograr una aproximación interseccional a la situación actual del sistema educativo nacionalista.

Para lograr una aproximación exitosa, se utiliza una narrativa desde una perspectiva comparativa de estas tres variables o reformas, las cuales son importantes para tener una lectura interseccional, pero que se deben entender como recíprocas.

\section{Devenir hacia la reforma económica del sistema educativo}

Esta se da con una transformación estructural en su régimen económico hegemónico de corte neoliberal, hacia un régimen económico más horizontal y sano o menos excluyente de las personas o comunidades menos privilegiadas.

En términos del régimen económico, las políticas de la corriente neoliberal hegemónicas que prevalecen en el país desde la década de 1980, han debilitado la estructura instituciona ${ }^{20}$ en diversos sectores como salud, cultura y educación; pilares de la formación de una ciudadanía crítica y consciente. Además, la crisis fiscal emergente de las últimas décadas, potencializada durante la crisis sanitaria del 2020 causada por el Covid-19, ha incrementado la dificultad de sostener la inversión fiscal que el país realiza para el financiamiento educativo.

El debilitamiento estructural del sistema educativo público produce situaciones emergentes que fomentan un ambiente tenso entre los principales actores del sistema, entre ellos: organizaciones gremiales, directores, docentes, administrativo, padres de

\footnotetext{
${ }^{19}$ Gerda Lerner, La creación del patriarcado (Barcelona: Editorial Crítica, 1986).

${ }^{20}$ David Díaz Arias, Historia del neoliberalismo en Costa Rica: la aparición en la contienda electoral, 1977-1978 (San José: Centro de Investigaciones Históricas de América Central de la UCR, 2019).
} 
familia y estudiantes, como lo manifestó la huelga magisterial del $2018^{21}$; quienes re-direccionan los esfuerzos institucionales y de los distintos actores mencionados a solucionar el problema de manera asistencialista, por lo tanto, imposibilita la capacidad de transformación y de mejorar la calidad del sistema educativo de forma estructural.

La corriente neoliberal reduce y erosiona la posibilidad de la organización social como respuesta y planteamiento de nuevas reformas políticas en beneficio de las clases más empobrecidas ${ }^{22}$, lo cual se ve reflejado en la precarización y pérdida de las garantías sociales de los trabajadores del sistema educativo público y en la ausencia de estas garantías para el sector privado. El neoliberalismo también promueve valores patriarcales ${ }^{23}$, tales como la competitividad entre las personas del cuerpo docente y las personas del cuerpo administrativo, obteniendo así espacios y relaciones laborales toxicas (como el exceso de carga administrativa y docente) que inciden en la salud emocional y mental.

Adicionalmente, urge atender la situación creciente de la deficiente formación, o deformación, universitaria de miles de docentes que se gradúan al año, a quienes se les relaciona directamente con las empresas de las universidades privadas ${ }^{24}$. Justamente, estas instituciones no invierten en extensión social e investigación, ambos aspectos considerados pilares de la educación universitaria modernizada, ya que ambos aspectos se consideran un gasto para los inversionistas de la educación privada, a menos de que se consiga lucrar de la práctica de alguna de ellas como fue mencionado en la primera parte de este texto; pero, al mismo tiempo, las universidades privadas son las mayores generadoras de profesionales en educación del país ${ }^{25}$, con el problema de que muchos de estos profesionales no cuentan con la formación y capacidad para responder a los objetivos de la política pública en educación ${ }^{26}$.

Bajo esta coyuntura, se entiende cómo la educación privada en primaria y secundaria ha tomado ventaja y se ha posicionado como la mejor opción, desde el privilegio, para la educación de miles de niños que tienen acceso a los recursos financieros y materiales para asistir al sistema educativo privado.

\section{Devenir hacia la reforma epistémica decolonial del sistema educativo}

El sistema educativo ha jugado un papel protagónico como herramienta para la homogeneización lingüística y cultural ${ }^{27}$ en la conformación de los Estados soberanos modernos hetero-capitalistas occidentalizados; asimismo, forma un sentido de identidad y ciudadanía basado en un discurso nacionalista excluyente, misógino y xenofóbico. En otros Estados soberanos modernos hetero-capitalistas occidentalizados de otras latitudes, este discurso legitimiza la presencia militar y las violentas guerras, ya que la reproducción de este modelo obstaculiza la posibilidad real de una reforma epistémica de su malla curricular a una forma de pensamiento

\footnotetext{
${ }^{21}$ «Ilegalidad de huelga en el MEP por no ser movimiento pacifico», Ministerio de Educación Pública, acceso: 9 de octubre de 2020, https://www.mep.go.cr/noticias/ilegalidad-huelga-mep-no-sermovimiento-pacifico

${ }^{22}$ Díaz Arias, Historia del neoliberalismo...

${ }^{23}$ Lerner, La creación del patriarcado...

${ }^{24}$ Programa Estado de la Nación, Sexto Estado de...

25 Ibíd.

${ }^{26}$ Ibíd.

${ }^{27}$ Jocelin Venegas Martinéz y Luz María Stella Moreno Medrano, «Breve panorama historico de la educacion intercultural en America Latina», DIDAC, n. ${ }^{\circ} 76$ (2020): 78.
} 
crítico y militante que deje de ser colonizador, jerárquico, dicotómico y complaciente con el régimen económico neoliberal.

El régimen neoliberal sabe cómo despolitizar estas formas subversivas para resignificarlas y abstraerlas de su fuerza subversiva. Por ejemplo, hace menos de 20 años la diversidad cultural se comienza a integrar en los planes de estudio, desde un lugar complaciente que no visibiliza la desigualdad y la violencia estructural que se arrastra en la región desde épocas de la Colonia, más detalladamente, se puede mencionar el cambio de llamar día del encuentro de razas en conmemoración del descubrimiento de América por parte de los colonos españoles. Lo que sugiere que esta reforma epistémica viene acompañada de una reforma etimológica de cómo trasmitir el conocimiento que incluya desde qué lugar se enuncia el conocimiento, cómo se enuncia y para quién se enuncia.

La construcción de una pedagogía crítica y militante es urgente, desde un lugar de reivindicación ${ }^{28}$ para las personas y poblaciones más marginalizadas por el Estado soberano moderno hetero-capitalista occidentalizado. Esto, permitirá plantear políticas públicas para re-dirigir e incluir una perspectiva más sensible hacia las diferencias culturales, sexuales, políticas e ideológicas de cualquier grupo de la población.

La reforma epistémica, etimológica de los saberes debe ser desde una perspectiva crítica, militante ${ }^{29}$ y no complaciente con los regímenes fundadores de los Estados soberanos modernos hetero-capitalistas occidentalizados. Esta reforma es la única posibilidad real de construirse de manera horizontal entre las distintas formas de ver y entender el mundo (indígenas, no indígenas, afrodescendientes, homosexuales o heterosexuales, de clase trabajadora o clase adinerada) ${ }^{30}$, al mismo tiempo que se posiciona en la búsqueda por la igualdad de derechos de los más desprotegidos, como es el caso de los pueblos indígenas, afrodescendientes, mujeres y personas de la diversidad sexual, entre muchas otras poblaciones invisibilizadas.

Esta reforma epistémica se ve materializada en los planes de estudio actuales, donde los 100 mejores centros educativos ${ }^{31}$ del país tienen un plan de estudio, aprobado por el Ministerio de Educación Pública (MEP), en el cual se ofrecen pedagogías alternativas con enfoques de pensamiento filosófico, humanista o científico; pero, 51 de estos 100 centros educativos siguen reproduciendo una corriente de pensamiento que llaman alternativa desde un enfoque o perspectiva hegemónica que es de base cristiana. Lo que sugiere que se sigue perpetuando un pensamiento que favorece prácticas y actitudes homofóbicas, machistas, racistas y misóginas en los estudiantes y el cuerpo docente; valores legitimados por el discurso cristiano.

La malla curricular del sistema educativo público ha tenido muy pocas reformas ${ }^{32}$ por la misma falta de recursos humanos, materiales y financieros. Al mismo tiempo que deben enfrentarse a situaciones que entorpecen el proceso al tratar de incluir de manera efectiva las pocas de estas reformas aprobadas en sus planes de estudio, el

\footnotetext{
${ }^{28}$ Venegas Martinéz y Moreno Medrano, «Breve panorama..., 78.

${ }^{29}$ Ibíd., 82.

${ }^{30}$ Ibíd.

${ }^{31}$ EKA, «Ranking de colegios 2019», La Revista empresarial, acceso: 18 de junio de 2020 , https://www.feriadecolegios.com/noticias-colegios/2020/9/22/los-100-mejores-colegios-privadossegn-bachillerato-del-mep

32 Programa Estado de la Nación, Resumen Séptimo Informa Estado de la Educación, San José: Masterlitto, 2019, https:/estadonacion.or.cr/wp-content/uploads/2019/08/Estado-

Educacio\%CC\%81n-RESUMEN-2019-WEB.pdf
} 
mejor ejemplo de esta situación son los programas o guías de afectividad y sexualidad del MEP, que publicó en el 2018.

\section{Devenir hacia la reforma organizativa del sistema educativo}

Es importante visibilizar el esfuerzo de la población que habitó durante el periodo de la construcción del Estado benefactor, principalmente por darle acceso a la educación mediante distintas formas de organización social. De esta manera, se logró que muchas comunidades, la mayoría de zonas rurales, obtuvieran los recursos, tanto materiales como financieros, para construir distintos centros educativos en distintas regiones del país.

Aun así, la red de centros educativos que existe actualmente solo abarca un $70 \%$ del territorio nacional, aproximadamente. En el país, existen alrededor de 4818 centros educativos de distintas categorías y modalidades: técnicos, científicos, humanistas y agropecuarios, entre otros; de ellos, aproximadamente el $10 \%$ (448) corresponde a instituciones privadas, mientras que solo un $2 \%$ se encuentra en la lista de los 100 mejores centros educativos del país.

Ya que la educación pública no tiene la misma cobertura y calidad en todo el territorio nacional, no llega a toda la ciudadanía en las mismas condiciones, como se mencionó en el primer texto publicado en Perú, donde se indica que el $60 \%$ de la población costarricense vive en condiciones de desigualdad estructural ${ }^{33}$, esto quiere decir que no tiene acceso a salud, trabajo y educación de calidad ${ }^{34}$. Por lo que la institucionalidad debe iniciar, con urgencia, un proceso de legitimación de diversas formas de enseñanza ${ }^{35}$ (pedagogía y metodología) que den acceso real a la educación a diversas poblaciones en todo el territorio nacional.

Sin duda existe un fuerte vallecentrismo ligado a las políticas neoliberales y a las formas de organización jerárquicas que fortalecen la región central del país, con ello se debilitan las zonas periféricas. De la lista de los mejores centros educativos del país, según la revista EKA empresarial, 21 se encuentran ubicadas en zonas periféricas fuera de la Gran Área Metropolitana (GAM), mientras que los 79 mejores centros educativos restantes se encuentran concentrados en la GAM.

Esta variable también está vinculada al espacio geográfico en el cual se encuentran ubicados los mejores centros educativos del país, existe una fuerte relación entre la clase social (según el índice de desarrollo humano del cantón y el lugar donde se encuentra el centro educativo). Los mejores centros educativos del país se encuentran en cantones con índices de desarrollo humano muy altos $^{36}$, por ejemplo: De las 79 mejores posiciones de la escala en el país, 44 (el 55\%) de los mejores centros educativos se encuentran en la provincia de San José, distribuidos en los cantones de Santa Ana, Escazú, Curridabat, Goicochea y Montes de Oca; mientras que el resto está en la provincia de Heredia, en los cantones de Belén, Santo Domingo y San Isidro. Todos ellos poseen índices de desarrollo humano superior a 0.87

\footnotetext{
${ }^{33}$ Luis Alonso Rojas Herra, «Apuntes hacia una crítica cuir del sistema de educacion pública en Costa Rica», Revista Disenso. Crítica y reflexión Latinoamericana 3, n. ${ }^{\circ} 2$ (2020).

${ }^{34}$ Joan Benach, Montse Vergara y Carles Muntaner. «Desigualdad en salud: la mayor epidemia del siglo XXI», Papeles de relaciones ecosociales y cambio global, n. ${ }^{\circ} 103$ (2008).

${ }^{35}$ Renata Cervetto y Miguel López, Agítese antes de usar: desplazamientos educativos, sociales y artísticos en América latina (Costa Rica: Teorética, 2017).

${ }^{36}$ Programa de las Naciones Unidas para el Desarrollo (PNUD), «Atlas de desarrollo Humano cantonal 2020 Costa Rica», acceso: 8 de febrero de 2021,

https://www.cr.undp.org/content/costarica/es/home/atlas-de-desarrollo-humano-cantonal.html
} 
(excepto Goicochea 0.82 ) e índices de educación superior a 0.82 (excepto Goicochea $0.79)$.

Por otro lado, los centros educativos categorizados con menor rendimiento se encuentran en distritos como Nacascolo, Culebra, Iguanita, Cañas Dulces, Curubande, Brasilito, Puerto Viejo, Matapalo, Nambí, Nicoya, Quirimancito, Upala, Las Delicias, El Roble, Barranca, Chacarita, Puntarenas, Herradura, Jaco, Quebrada Ganado, Tarcóles, Parrita, Quepos, Naranjito, San Isidro del General, Puerto Viejo de Talamanca, Talamanca, Bribri, Manzanillo, Cahuita, Limón, Parismina, Matina, Batán, Pacuarito, Siquirres, Guápiles, Pacora, Pacayas, Cervantes, Aguas Zarcas, Buenos Aires y San Carlos. En todos ellos se puede encontrar escuelas y colegios localizados en las zonas con el menor índice de desarrollo humano del país ${ }^{37}$, inferiores a 0.80 y con índices de educación inferiores a 0.75 , distribuidos en los cantones de Nicoya, Nandayure, Santa Cruz, Hojancha, Parrita, Garabito, Upala, Siquirres y Talamanca.

En conjunto, conforman los centros de población periférica más empobrecidos y vulnerabilizados del país. Además, si se analiza en comparación los mapas 3.2 y 4.1 (siguiente página) del Séptimo Informe del Estado de la Educación Costarricense del 2019, en las páginas 86 y130 respectivamente, se evidencia la fuerte relación que produce la presencia de narcotráfico en esas comunidades mencionadas ${ }^{38}$, ya que el mayor indicador en el índice de exclusión educativa que se encuentra asociado a estos espacios territoriales es la posesión de droga, seguido por la pobreza.

Los cambios políticos y en lo económico (formal e informal) inciden particularmente en las formas de enseñanza en las poblaciones de las distintas porciones del territorio; con ello, se manifiestan situaciones pedagógicas y metodológicas particulares en cada uno; esto sería una lectura desde la territorialización ${ }^{39}$, por lo que el sistema educativo debe reestructurar sus formas de trasmitir y producir el conocimiento de una estructura homogénea y rígida a una estructura versátil y fluida desde la empatía por las distintas realidades ${ }^{40}$ de la población costarricense.

Por ejemplo, en los tiempos de desplazamiento, la mayoría de los estudiantes del Valle Central, en promedio, invierten una hora para desplazarse al centro educativo al cual asisten. Mientras que en zonas rurales el tiempo se duplica, hasta llegar a casos extremos donde un estudiante de territorio indígena invierte hasta cuatro horas de desplazamiento, caminando (cuando la ruta se encuentra en buenas condiciones), para llegar al centro educativo más cercano y otras cuatro horas para volver a su casa $^{41}$.

\footnotetext{
37 Íbid.

${ }^{38}$ Organización de Estados Americanos (OEA), «Informe sobre el consumo de drogas en las Américas 2019», acceso: 9 de octubre de 2020,

http://www.cicad.oas.org/oid/pubs/Informe $\% 20$ sobre $\% 20 \mathrm{el} \% 20$ consumo $\% 20 \mathrm{de} \% 20$ drogas $\% 20 \mathrm{en} \% 2$ 0las\%20Am\%C3\%A9ricas\%202019.pdf

${ }^{39}$ Luis M. Barboza-Arias, Karen Chacón-Araya y Daniel Alpzar-Rojas, «Narrativas de territorialización en las políticas públicas en Costa Rica y sus efectos en una comunidad indígena», Eutopía, revista de desarrollo económico territorial, n. ${ }^{\circ} 18$ (2020): 49.

${ }^{40}$ Cervetto y López, Agítese antes de usar...

${ }^{41}$ «Informe general proyecto de los talleres participativos para los 13 centros educativos del MEP en zonas indígenas» Larh arquitectura e investigación del paisaje social. Acceso: 26 de enero de 2021, https://larharquitectura.wordpress.com/2018/02/19/proyecto-de-los-talleres-participativos-para-los13-centros-educativos-del-m-e-p-en-zonas-indigenas/
} 


\section{Mapas 3.2 y 4.1 del Séptimo informe del Estado de la Educación Costarricense del 2019}
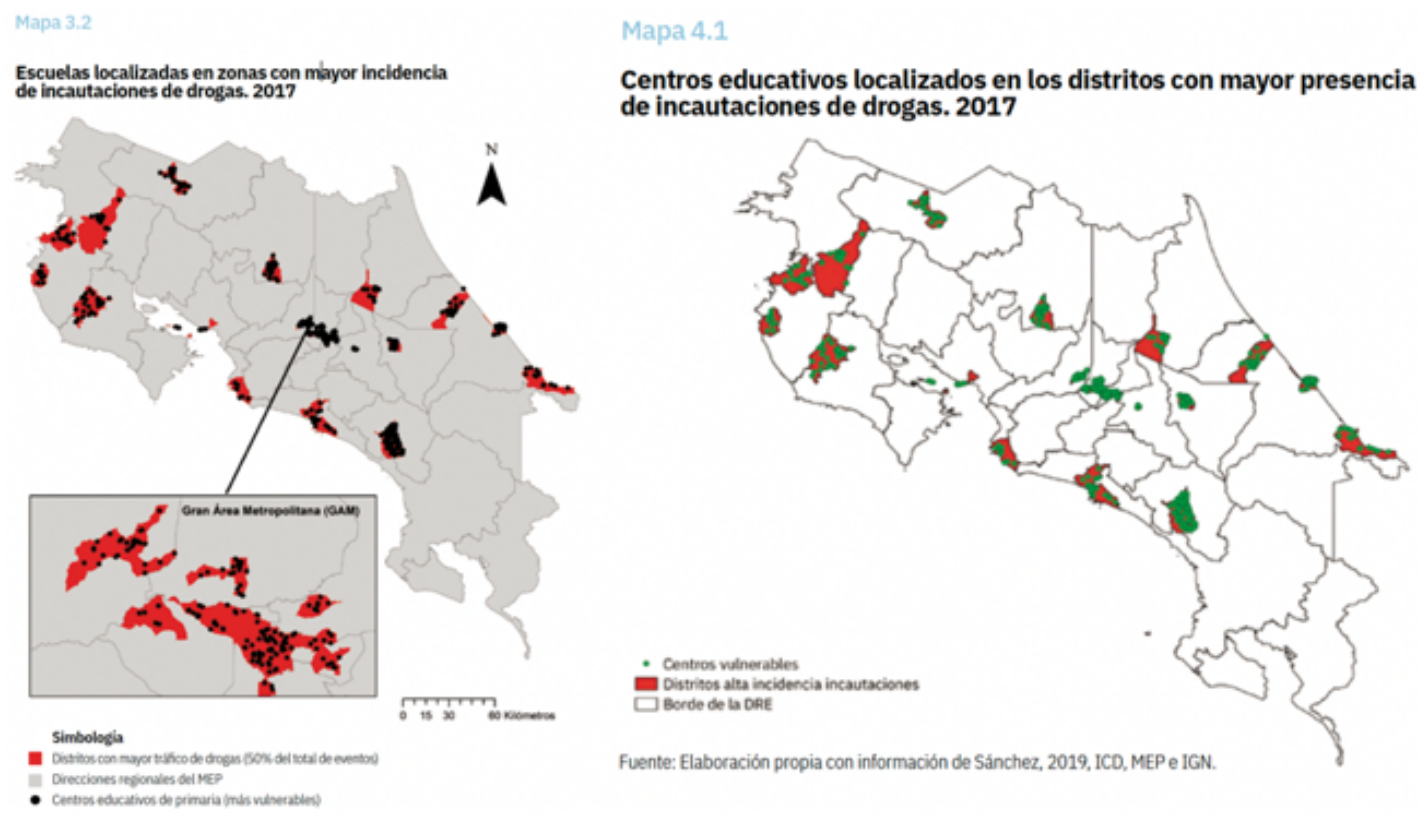

Fuente: Estado de la Educación Costarricense, 2019.

Cuando el sistema educativo impulsa iniciativas de carácter público de manea homogénea desde el privilegió vallecentralista, no toma en consideración las situaciones particulares emergentes de cada territorio. En 2017, durante el cierre del puente de la platina, el MEP desarrolló un protocolo en el cual se le permitió llegar tarde al cuerpo docente y a los estudiantes del Valle Central; pero, en territorio indígena, los docentes y estudiantes no cuentan con protocolos que justifiquen la llegada tardía o la ausencia del docente en casos de deslizamientos o inundaciones, las cuales son frecuentes en esas zonas.

Por último, la calidad espacial del diseño arquitectónico de los centros del sistema educativo del país, se ve afectada por la crisis fiscal, la cual imposibilita el autofinanciamiento de la infraestructura arquitectónica para todo el territorio. Mientras que en los países desarrollados se evalúa la calidad del espacio y mobiliario, produciendo tipologías arquitectónicas educativas vanguardistas que incorporan la perspectiva de metodologías y enfoques pedagógicos alternativos, Costa Rica se enfrenta a un sistema de contratación pública (burocratizado) para el sistema educativo de este sector que favorece las licitaciones que propongan propuestas lo más rápido y barato posible. Se debe recordar que el precio y el plazo son los requisitos con mayor peso o puntaje en las licitaciones públicas, lo cual deja en evidencia que estas no están en función de la calidad de los espacios 
arquitectónicos $^{42}$ para favorecer el proceso de enseñanza de las personas que los habitan.

\section{Des-conclusiones: hacia un devenir trans-educativo}

La Constitución Política, como toda construcción social, con el pasar del tiempo se va desgastando. Esta es la razón por la cual se necesita aplicar reformas de carácter estructural que, de manera afectiva (empatía) y efectiva, solventen las grandes desigualdades que prevalecen en los países de la región, organizados bajo el modelo de Estado soberano occidentalizado. Porque, si bien la Constitución no es la solución a todos problemas del sistema educativo, ningún problema se puede resolver al margen de ella.

Por esto, las respuestas generadas por el sistema educativo para solventar estos déficits deben ser desde la empatía ${ }^{43}$ con los grupos menos privilegiados de la región, posibilitando la autonomía y la representatividad desde la reivindicación de estos grupos en las políticas nacionales para cualquier reforma de sus regímenes fundacionales incluyendo la educativa.

Por otro lado, desde una perspectiva financiera de un orden económico neoliberal globalizado, las políticas de este corte presionan a que cada vez sea más necesario el financiamiento internacional para el funcionamiento de las instituciones públicas. Para esto, las instituciones se ven envueltas en un ambiente fuertemente competitivo, donde son evaluadas por procesos internacionales.

Los indicadores para medir el estado de la educación en general tienen una perspectiva técnica y financiera, la cual deja de lado una serie de variables sociaafectivas que urge el involucramiento en estos parámetros. Los indicadores actuales miden la cantidad de personas matriculadas, el gasto económico anual, así como el llamado «clima educativo el hogar», que se mide por los ingresos económicos de la familia. Para tener una aproximación, algunas de estas herramientas de medición que se indican en el Sexto Informe del Estado Nación de la Educación ${ }^{44}$ son:

- Instrumento Class.

- Woodcock-Muñoz.

- Pruebas PISA (2015).

- Sistema Nacional de Acreditación de la Educación Superior (Sinaes).

- Escala Ecers-R, un instrumento útil para evaluar la calidad de la educación, conformada por siete subescalas.

- Pruebas Fortalecimiento de aprendizajes para la renovación de oportunidades (FARO), aprobadas por el Consejo Superior de Educación (CSE) en 2019.

La mayoría de las herramientas de medición son de orden colonial, estas son efectivas para medir los estándares educativos en los países de Estados soberanos

\footnotetext{
42 «Programa galería urbana: Centros educativos públicos: ¿hemos mejorado en su diseño y adecuación al entorno?», CARCR, acceso: 26 de enero de 2021, https://www.facebook.com/CACRarquitectos/videos/1137362490048202

${ }^{43}$ Benach, Vergara y Muntaner, 2008, Desigualdad en salud...

${ }^{44}$ Programa Estado de la Nación, Sexto Estado de...
} 
desarrollados, pero olvidan que los países latinoamericanos arrastran una condición de desigualad estructural desde la constitución del Estado soberano, en el caso de esta región, hace 200 años no se ha logrado superar esta problemática. Además, casi no hay instrumentos de monitoreo y seguimiento que le permitan dar una continuidad a los procesos que estos instrumentos de medición categorizan.

La brecha de desigualdad social entre Estados desarrollados y Estados en vías de desarrollo es gigante. No es ético medir el sistema educativo nacional con las mismas normas y estándares que lo hacen en los países de Europa Occidental o Estados Unidos.

La conmemoración del bicentenario debe ser un espacio para la reflexión desde un lugar critico sobre los regímenes fundacionales que construyen socialmente las nociones de Estado, ciudadanía y territorio, entre otras. Este ejercicio epistémico en todas las escalas del aparato institucional posibilitará y contribuirá a la transformación a una democracia más sana, inclusiva y que valore positivamente lo diverso dentro de su rígida estructura estatal actual.

\section{Formato de citación según APA}

Rojas-Herra, L. A. (2021). Apuntes hacia una crítica cuir del sistema de educación pública en Costa Rica (parte II). Revista Espiga, 20 (42), 37-49.

Formato de citación según Chicago-Deusto

Rojas-Herra, Luis Alonso. «Apuntes hacia una crítica cuir del sistema de educación pública en Costa Rica (parte II)». Revista Espiga 20, n. ${ }^{\circ} 42$

(setiembre, 2021): 37-49. 


\section{Referencias}

Barboza-Arias, Luis M., Karen Chacón-Araya y Daniel Alpizar-Rojas, «Narrativas de territorialización en las políticas públicas en Costa Rica y sus efectos en una comunidad indígena». Eutopía, revista de desarrollo económico territorial, n. $^{\circ} 18$ (2020): 49-69.

https://revistas.flacsoandes.edu.ec/eutopia/article/view/4639

Benach, Joan, Montse Vergara y Carles Muntaner. «Desigualdad en salud: la mayor epidemia del siglo XXI». Papeles de relaciones ecosociales y cambio global, n. ${ }^{\circ} 103$ (2008): 29-40.

https://www.fuhem.es/papeles_articulo/desigualdad-en-salud-la-mayorepidemia-del-siglo-xxi/

CARCR. «Programa galería urbana: Centros educativos públicos: ¿hemos mejorado en su diseño y adecuación al entorno?». Acceso: 26 de enero del 2021.

https://www.facebook.com/CACRarquitectos/videos/1137362490048202

Cervetto, Renata y Miguel López. Agitese antes de usar: desplazamientos educativos, sociales y artísticos en América latina. San José: Teorética, 2017.

Díaz Arias, David. Historia del neoliberalismo en Costa Rica: la aparición en la contienda electoral, 1977-1978. San José: Centro de Investigaciones Históricas de América Central de la UCR, 2019.

EKA. «Ranking de colegios 2019». La Revista empresarial. Acceso: 18 de junio de 2020. https://www.feriadecolegios.com/noticias-colegios/2020/9/22/los-100mejores-colegios-privados-segn-bachillerato-del-mep

Falconí Trávez Diego, Santiago Castellanos y María Amelia Viteri, ed. Resentir lo queer en América Latina: diálogos desde / con el sur. España: editorial Egales, 2014.

Falconí Trávez, Diego, ed. Inflexión marica, escrituras del descalabro gay en América Latina. España: editorial Egales, 2018.

Larh arquitectura e investigación del paisaje social. «Informe general proyecto de los talleres participativos para los 13 centros educativos del MEP en zonas indígenas». Acceso: 26 de enero de 2021.

https://larharquitectura.wordpress.com/2018/02/19/proyecto-de-los-talleresparticipativos-para-los-13-centros-educativos-del-m-e-p-en-zonas-indigenas/

Lerner, Gerda. La creación del patriarcado. Barcelona: Editorial Crítica, 1986.

Martínez Gutiérrez, Bernal. Cronología de la educación costarricense. Costa Rica: Imprenta Nacional, 2016. 
Ministerio de Educación Pública (MEP). «Ilegalidad de huelga en el MEP por no ser movimiento pacífico». Acceso: 9 de octubre de 2020.

https://www.mep.go.cr/noticias/ilegalidad-huelga-mep-no-ser-movimientopacifico

Organización de Estados Americanos (OEA). «Informe sobre el consumo de drogas en las Américas 2019». Acceso: 9 de octubre de 2020.

http://www.cicad.oas.org/oid/pubs/Informe\%20sobre $\% 20 \mathrm{el} \% 20$ consumo $\% 20 \mathrm{de} \% 2$ 0drogas\%20en\%201as\%20Am\%C3\%A9ricas\%202019.pdf

Organización de Estados Americanos (OEA). «Informe sobre el consumo de drogas en las Américas 2019». Acceso: 9 de octubre de 2020.

http://www.cicad.oas.org/oid/pubs/Informe\%20sobre $\% 20 \mathrm{el} \% 20$ consumo $\% 2$ 0de\%20drogas\%20en\%20las\%20Am\%C3\%A9ricas\%202019.pdf

Programa de las Naciones Unidas para el Desarrollo (PNUD). «Atlas de desarrollo Humano cantonal 2020 Costa Rica». Acceso: 8 de febrero de 2021. https://www.cr.undp.org/content/costarica/es/home/atlas-de-desarrollohumano-cantonal.html

Programa Estado de la Nación. Sexto Informe Estado de la Educación. San José: Servicios Gráficos, A. C., 2017. Recuperado de https:/www.estadonacion.or.cr/educacion2017/assets/ee6-informecompleto.pdf

Programa Estado de la Nación. Resumen Séptimo Informe Estado de la Educación. San José: Masterlitto, 2019.

Rojas Herra, Luis Alonso, «Apuntes hacia una crítica cuir del sistema de educación pública en Costa Rica». Revista Disenso. Crítica y reflexión Latinoamericana, Vol. 3, n. ${ }^{\circ} 2$ (2020): 48-61. https://barropensativo.com/index.php/DISENSO/article/view/85

Solá, Miriam y Elena Urko. Transfeminismos epistemes, fricciones y flujos. Navarra: Ediciones Txalaparta, 2018.

Venegas Martínez, Jocelin y Luz María Stella Moreno Medrano. Breve panorama histórico de la educación intercultural en América Latina. DIDAC, ${ }^{\circ}{ }^{\circ} 76$ (2020): 77-85. 